${ }^{1}$ Departamento de Ciencias Neurológicas Oriente, Facultad de Medicina, Universidad de Chile, Santiago.

${ }^{2}$ Servicio de Neurología, Hospital Salvador, Providencia, Santiago.

Recibido el 14 de agosto de 2014, aceptado el 21 de enero de 2015.

Los resultados de este trabajo han sido publicados parcialmente como resumen de congreso en la revista de la Sociedad Chilena de Neurología, Psiquiatría y Neurocirugía: Cea G, Jiménez D. Distrofia fascioescapulohumeral: Presentación de una serie con estudio genético. Revista Chilena de Neuro-Psiquiatría. 2012; 50 (supl 1): 7.

El trabajo fue realizado sin el requerimiento de ayudas o fuente de financiamiento. Los autores no tienen conflicto de intereses que declarar.

Correspondencia a: Gabriel Cea

Servicio de Neurología, Hospital del Salvador.

Avenida Salvador 364,

Providencia, Santiago, Chile. Celular: 76597055 jcea@med.uchile.cl

\section{Distrofia muscular facioescapulohumeral en Chile: presentación de serie en hospital de referencia terciario}

\author{
GABRIEL CEA ${ }^{1,2}$, DANIEL JIMÉNEZ ${ }^{2}$
}

Background: Facioscapulohumeral muscular dystrophy is the third most common muscular dystrophy with an estimated prevalence of 1 per 20.000 and a normal life expectancy in the majority of patients. However, approximately $15 \%$ of patients become wheelchair bound in the course of their life. It is a hereditary autosomal dominant disease with high (95\%) penetrance by the age of 20, but with variable degree of phenotypic expression even in the same family group. Symptoms frequently start in the second decade of life, with facial and scapular weakness. Aim: To report the clinical features of seven patients with the disease, seen at a public hospital. Material and Methods: Analysis of seven patients with genetic study seen in a public Hospital in Santiago. Results: The age of patients fluctuated from 18 to 61 years and four were females. The mean age at onset of symptoms was 29 years and four had a family history of the disease. The usual presenting complaint was arm or shoulder asymmetric weakness. Four patients had bone pain. Facial involvement was present in four. A genetic study was done in five patients, the other two patients were relatives, confirming the contraction or lower number of repetitions in D4Z4 region. After 12 years of follow up only 2 patients older than 60 years cannot work and one female patients is in a semi dependent state at the age of 30. Conclusions: The clinical workup in the diagnosis and the timely indication of genetic studies are highlighted, to avoid unnecessary and invasive procedures. The variability in the phenotypic expression in a similar genetic defect is discussed and the genetic or epigenetic mechanisms of this muscular dystrophy are described.

(Rev Med Chile 2015; 143: 304-309)

Key words: Chile; Muscular diseases; Muscular dystrophy, facioscapulohumeral.

$\mathrm{D}$ e acuerdo a los escasos estudios epidemiológicos al respecto, se estima que la distrofia muscular facioescapulohumeral (DMFEH) tiene una prevalencia de 1 por $20.000^{1,2}$. Aunque cerca de un 15\% de los afectados llega a depender de una silla de ruedas, la mayoría de los pacientes logran desarrollar una vida bastante normal y en algunas series de casos se describe hasta un $30 \%$ de pacientes asintomáticos ${ }^{1}$. Característicamente, afecta de manera asimétrica, primero los músculos de la cara y la cintura escapular, seguido por debilidad de los músculos peroneos y de la cintura pelviana. Las manifestaciones extramusculares son raras, siendo las más comunes la pérdida auditiva y las telangectasias retinales, mientras que el compromiso cardiaco es infre- 
cuente ${ }^{3-5}$. Su herencia es autosómica dominante, con una alta penetrancia (95\%) a los 20 años, pero con una expresividad fenotípica variable, incluso en miembros de una misma familia ${ }^{6,7}$.

El defecto genético se ubica en D4Z4 en la región subtelomérica del cromosoma 4q35 y consiste en una contracción de repeticiones en esta zona, que normalmente fluctúa entre 11 y 100 repeticiones. La aparición de síntomas se produce cuando la contracción es de 10 o menos repeticiones de $3,3 \mathrm{~kb}^{8,9}$. La contracción de D4Z4 no siempre es patogénica y necesita de ciertas condiciones de "permisividad" de la región 4q35, que permiten la poliadenilación y transcripción del homeobox DUX4. La acumulación de este y otros transcriptos tendrían un efecto tóxico sobre el músculo y otros tejidos ${ }^{10,11}$.

Hasta donde sabemos no existen series publicadas en Chile sobre esta condición y consideramos que es necesario entregar esta información para que tengamos más herramientas clínicas que permitan reconocer esta enfermedad en forma oportuna. Se presenta una serie de pacientes evaluados en el Servicio de Neurología del Hospital del Salvador en quienes se ha establecido el diagnóstico, confirmado por estudio genético. Se enfatiza la variabilidad clínica y el subdiagnóstico y se discuten los mecanismos genéticos involucrados en la etiopatogenia.

\section{Método}

Desde el año 1985 a la fecha, nuestro centro ha mantenido una base de datos con 7 pacientes con DMFEH, que se han mantenido en control con distinta frecuencia de acuerdo a su evolución clínica. El análisis de cada paciente se hizo en base a una pauta de evaluación de los segmentos corporales involucrados, teniendo en cuenta la atrofia, debilidad y asimetría de estos. También se consideró las dificultades o problemas que los pacientes presentaban en su vida diaria. Este estudio se hizo de acuerdo a las normas de investigación en seres humanos y contó con la aprobación del Comité de Ética Científico del Servicio de Salud Metropolitano Oriente.

El estudio genético se realizó en Bristol, Reino Unido, con excepción del paciente 6 que se realizó en la Clínica Mayo, Estados Unidos de Norteamérica. Este estudio genético se realiza con una digestión doble EcoRI/Blnl del DNA del paciente, que iden- tifica un fragmento indicativo de un rearreglo en el cromosoma $4 \mathrm{q} 35 \mathrm{y}$, que al mismo tiempo, elimina otras estructuras similares en el cromosoma 10q26 y en el cromosoma Y. Este defecto está presente en más del 95\% de los casos de distrofia DMFEH ${ }^{12,13}$.

\section{Resultados}

La Tabla 1 presenta las características clínicas y de laboratorio más importantes de nuestra serie. Se controlaron 7 pacientes ( 4 mujeres y 3 hombres), con un promedio de edad de 39,4 años (rango 18-61 años) al momento de consultar por primera vez. La edad promedio de inicio de los síntomas fue de 29,1 años y el tiempo promedio entre el inicio de los síntomas y la primera consulta fue de 10,2 años. En cuatro de nuestros pacientes existen antecedentes familiares de la enfermedad. Los pacientes 3 y 5 incluidos en la serie son hermanos, pero desconocen historia familiar porque sus padres inmigraron a Chile y perdieron contacto con el resto de la familia. El paciente 7 es hijo de la paciente 2, y ambos tienen historia familiar de la enfermedad. Los restantes tres pacientes no tenían historia familiar.

El motivo de consulta más común fue la debilidad de hombros o brazos. Sin embargo, en dos de nuestros pacientes la queja principal afectaba las extremidades inferiores. La debilidad se acompañó de dolor osteo-articular en cuatro de los casos. En el examen neurológico inicial todos los pacientes presentaban algún grado de debilidad en hombros y brazos, mientras que el compromiso facial era evidente en cuatro de los siete pacientes. En seis pacientes se pesquisó debilidad de tronco, que producía una hiperlordosis notoria. Respecto al compromiso de las extremidades inferiores al momento de la primera consulta, existía debilidad de la cintura pélvica en 2 de nuestros pacientes, debilidad de los extensores del pie en 3 de ellos, mientras que ningún paciente de la serie mostraba debilidad en muslos y pantorrillas (Figura 1). En todos los pacientes había un componente asimétrico en al menos uno de los segmentos corporales afectados al momento de consultar (Figura 2).

En el estudio inicial, se documentó una creatinfosfoquinasa (CPK) plasmática levemente elevada en los pacientes 1 y 2 . En estos dos pacientes se realizó EMG, revelando un patrón miopático en ambos casos. Dos de los casos (paciente 2 y 3 de la serie) fueron sometidos a una biopsia muscular. 
Tabla 1. Características clínicas y de laboratorio de 7 pacientes con diagnóstico de DMFEH

\begin{tabular}{|c|c|c|c|c|c|c|c|c|c|c|}
\hline $\mathbf{n}$ & Sexo & $\begin{array}{l}\text { Edad } \\
\text { inicio }\end{array}$ & $\begin{array}{l}\text { Edad de } \\
\text { consulta }\end{array}$ & $\begin{array}{l}\text { Motivo de } \\
\text { consulta }\end{array}$ & $\begin{array}{l}\text { Presentación }{ }^{1} \\
\text { clínica }\end{array}$ & $\begin{array}{c}\text { Asi- } \\
\text { metría }\end{array}$ & $\begin{array}{l}\text { Historia } \\
\text { familiar }\end{array}$ & EMG & CK & $\mathrm{D}^{2} 4^{2}$ \\
\hline 1 & $\mathrm{~F}$ & 23 & 24 & $\begin{array}{l}\text { Dolor pie derecho } \\
\text { y dificultad en la } \\
\text { marcha }\end{array}$ & $\mathrm{F}, \mathrm{H}, \mathrm{B}, \mathrm{T}, \mathrm{C}, \mathrm{E}$ & sí & No & $\begin{array}{l}\text { Patrón } \\
\text { miopático }\end{array}$ & 395 & 8 \\
\hline 2 & $\mathrm{~F}$ & 13 & 44 & $\begin{array}{l}\text { Debilidad de } \\
\text { ambos brazos }\end{array}$ & $\mathrm{F}, \mathrm{H}, \mathrm{B}, \mathrm{T}$ & sí & $\begin{array}{c}\text { Padre, abuelo, } \\
\text { tía y prima }\end{array}$ & $\begin{array}{l}\text { Patrón } \\
\text { miopático }\end{array}$ & 87 & 7 \\
\hline 3 & $\mathrm{~F}$ & 59 & 60 & $\begin{array}{l}\text { Dolor y debilidad } \\
\text { hombro derecho }\end{array}$ & $F, H, B, T, C$ & sí & $\begin{array}{l}\text { Hermana de } \\
\text { paciente } 5\end{array}$ & NR & ND & 10 \\
\hline 4 & $\mathrm{~F}$ & 10 & 36 & $\begin{array}{l}\text { Dificultad para ele- } \\
\text { var ambos brazos }\end{array}$ & $H, B, T, E$ & sí & No & NR & ND & 8 \\
\hline 5 & M & 55 & 61 & $\begin{array}{l}\text { Caída del pie } \\
\text { derecho }\end{array}$ & $\mathrm{H}, \mathrm{B}, \mathrm{T}, \mathrm{E}$ & sí & $\begin{array}{l}\text { Hermano de } \\
\text { paciente } 3\end{array}$ & NR & 174 & NR \\
\hline 6 & M & 15 & 18 & $\begin{array}{l}\text { Dolor hombro } \\
\text { derecho }\end{array}$ & $\mathrm{H}, \mathrm{B}$ & sí & No & NR & ND & 10 \\
\hline 7 & M & 29 & 33 & $\begin{array}{l}\text { Dolor y debili- } \\
\text { dad de ambos } \\
\text { hombros }\end{array}$ & $F, H, B, T$ & sí & $\begin{array}{l}\text { Hijo de pa- } \\
\text { ciente } 2\end{array}$ & NR & 158 & NR \\
\hline
\end{tabular}

${ }^{1} \mathrm{~F}$ : facial; H: hombro; B: brazo, T: tronco; C: cadera; M: muslo; P: pantorrilla; E: extensores del pie. ${ }^{2}$ Fragmento de contracción en región D4Z4 (doble digestión). ND: no disponible. NR: no realizado.

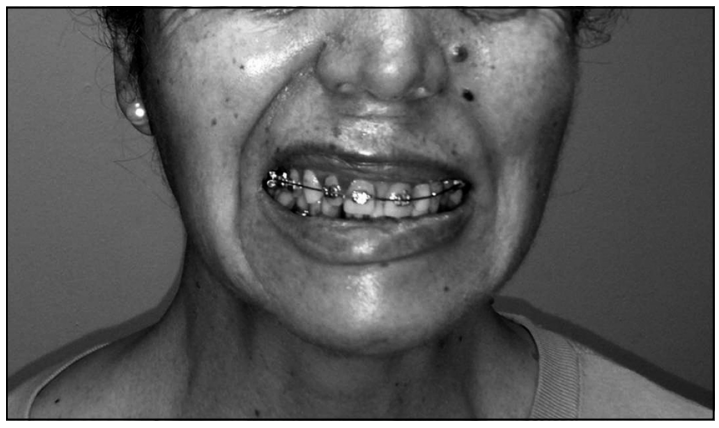

Figura 1. Fotografía de la paciente 2 que muestra una debilidad facial asimétrica presente al momento de consultar.

En el caso 3 el informe fue normal, mientras que en el caso 2 se informó una miositis. Por este motivo, la paciente 2 recibió tratamiento corticoidal por un período de varios meses hasta que se planteó el diagnóstico DFEH, el que posteriormente fue confirmado por estudio genético.

El estudio genético se realizó en 5 pacientes. En todos ellos confirmó una contracción o disminución de repeticiones, entre 7 y 10, en la región D4Z4. Los dos pacientes en quienes no se realizó el estudio son familiares directos de otros pacientes de la serie con diagnóstico confirmado.

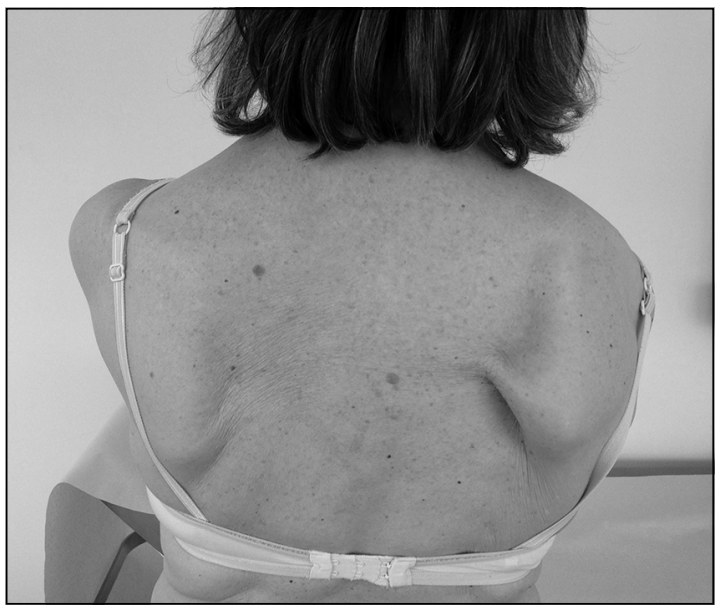

Figura 2. Marcada escápula alada derecha en la paciente 3, mostrando la típica asimetría en la presentación clínica de la DMFEH.

Los pacientes han sido seguidos por 12,2 años en promedio y la mayoría no ha presentado un compromiso funcional importante y son autovalentes. Dos pacientes mayores de 60 años (casos 2 y 4 ) ya no pueden trabajar. Sólo la paciente 1 ha empeorado hasta un estado de semi-dependencia a la edad de 30 años. 
Se presentan a continuación los casos de los paciente 1 y 2 de la serie para ejemplificar la variedad clínica y las dificultades diagnósticas que este cuadro puede tener:

\section{Caso 1}

Mujer de 24 años, sin antecedentes mórbidos personales ni familiares relevantes. Consulta por 1 año de evolución de dificultad progresiva de la marcha por debilidad y dolor del pie derecho. En el examen motor de ese momento destacaba la presencia de debilidad facial bilateral, escápula alada bilateral, debilidad leve y simétrica de bíceps y pectorales, debilidad en la dorsiflexión de ambos pies mayor a derecha y debilidad leve en la flexión de ambas caderas con fuerza conservada en cuádriceps y pantorrillas. Destaca una notoria atrofia de los dorsiflexores del pie, mayor a derecha. Presenta además leve escoliosis e hiperlordosis. Sin otros signos neurológicos.

El valor de CPK plasmática resultó levemente elevado (395 UI/L) y la electromiografía informó un patrón miopático. El estudio genético confirmó el diagnóstico de DMFEH, con una reducción a 8 repeticiones en la región $\mathrm{D} 4 \mathrm{Z} 4$.

Tras 12 años de evolución, se compromete progresivamente su funcionalidad hasta llegar actualmente a un estado de semi-autovalencia, requiriendo incluso ayuda para caminar. Durante este tiempo se ha desempeñado como secretaria y tuvo una hija sin evidencias de la enfermedad hasta el momento, que no ha sido evaluada por nosotros.

\section{Caso 2}

Mujer de 44 años, con antecedente de padre con diagnóstico de DMFEH. Consulta por debilidad de ambas extremidades superiores de un año de evolución, aunque en su adolescencia había notado una leve asimetría escapular y facial. En el examen motor, al momento de consulta, destacaba debilidad facial mayor a izquierda (Figura 3); atrofia y debilidad de la cintura escapular y de extremidades superiores, más notoria a izquierda, y una hiperlordosis. No existía atrofia ni debilidad en las extremidades inferiores.

En el estudio, la CPK plasmática resultó normal y la EMG mostró un patrón miopático. En esta paciente se realizó biopsia muscular del deltoides cuyo resultado fue concordante con una miositis. La paciente fue tratada con corticoides por varios meses hasta que fue reevaluada por nosotros. El estudio genético mostró una reducción a 7 repeticiones en la región D4Z4.

Tras 16 años de seguimiento, la paciente es independiente en las actividades de la vida diaria, aunque ha debido abandonar su actividad laboral, como asesora del hogar, desde hace un año. La paciente tuvo 3 hijos y uno de ellos, paciente 7 de la serie, quien nota los primeros síntomas típicos de la enfermedad a los 29 años.

\section{Discusión}

Nuestra serie es discreta pero hasta donde sabemos, constituye la primera publicada en Chile. Su importancia radica en que como todos los pacientes tienen un defecto genético definido, un seguimiento de largo plazo permite analizar la evolución del fenotipo. Cuatro pacientes relataron las molestias dolorosas como el motivo que los llevó a consultar, aunque sólo uno de ellos no había notado debilidad muscular. Tal vez este hecho clínico, la poca repercusión en la funcionalidad que experimentan la mayoría de los pacientes explique porqué tenemos una población de pacientes en control que no se corresponde con la prevalencia mundial, que sería de 1 por $20.000^{1,2}$. El desconocimiento del cuadro por pacientes y médicos puede ser otro factor que determina la

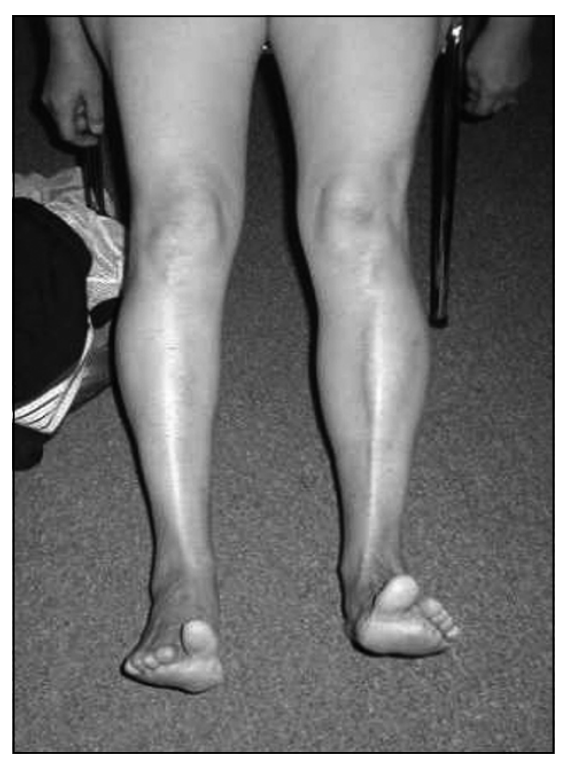

Figura 3. Fotografía que muestra la atrofia asimétrica de los extensores del pie en la paciente 4 de la serie. 
baja prevalencia observada en nuestro hospital. Por estas razones, creemos que esta presentación puede alertar sobre el importante sub-diagnóstico que esta enfermedad tiene en Chile.

En nuestra serie cuatro pacientes tenían antecedentes familiares y tres eran el primer caso en la familia, constituyendo una mutación nueva, que sería una proporción de mutaciones nuevas similar a lo que ha sido descrito en otras series más extensas ${ }^{14,15}$. Las mutaciones de novo fueron inicialmente descritas como raras en publicaciones más antiguas, pero no parece ser el caso en la epidemiología de la distrofia facioescapulohumeral ${ }^{16}$.

La evolución de los 2 casos descritos en detalle, ilustra que la severidad del fenotipo no siempre está en directa relación con la severidad del defecto genético. La primera paciente presenta un cuadro clínico severo, que le impone importantes limitaciones en su vida diaria a pesar de ser mucho más joven y tener un defecto genético menos severo que la segunda paciente, quien a los 60 años presenta mínimas restricciones en las actividades de la vida diaria. En la mayoría de los pacientes se observa una correlación entre la severidad de la restricción y la gravedad del fenotipo, pero existen excepciones en la expresión fenotípica incluso en familias con el mismo genotipo ${ }^{6,17,18}$. Por otra parte, existen algunas publicaciones donde se plantea un fenómeno de anticipación, o aumento de la severidad de la enfermedad de una generación a otra ${ }^{15,17}$. Sin embargo, este fenómeno no aparece descrito en la mayoría de las series publicadas en la literatura mundial, y en el único caso donde tenemos antecedentes en nuestra serie observamos que, el hijo de la paciente 2 tiene una edad de presentación 10 años más tardía.

La paciente 2 fue sometida a una biopsia muscular que mostró acúmulos inflamatorios, lo que condujo erróneamente al diagnóstico de polimiositis y tratamiento con prednisona por varios meses. La ocurrencia de fenómenos inflamatorios similares a una miositis es un aspecto que está descrito en la literatura y que no es infrecuente en $\mathrm{DMFEH}^{19,20}$. Esto plantea, además, que en esta como en otras enfermedades musculares es necesario cambiar procedimientos rutinarios que no prestan ayuda en el proceso diagnóstico y pueden enturbiarlo. Existe varias distrofias donde después de una buena evaluación se debe proceder al estudio genético, que debiera estar al alcance de estos pacientes y que es esencial para un adecuado consejo genético. Nuestro caso ilustra como una buena caracterización clínica terminó en el diagnóstico correcto y enmendó un error con potenciales consecuencias deletéreas para el paciente. Estudios como la electromiografía o la biopsia en este tipo de patologías musculares tienen un rol cuando el estudio genético no confirma el diagnóstico clínico y es necesario replantear el caso ${ }^{21}$.

Respecto al diagnóstico al clínico en la DMFEH se debe enfatizar el carácter típicamente asimétrico de la debilidad y que esto es una clave sustancial en el diagnóstico diferencial. La debilidad y atrofia pueden iniciarse en la cara, escápula, bíceps braquial o dorsiflexores del pie, pero la asimetría es su sello. Esto puede variar incluso en una misma familia, como ocurrió en nuestra serie: paciente 3 se presentó con escápula alada derecha y paciente 5 con debilidad en dorsiflexión del pie derecho. La edad de inicio es difícil de establecer, por lo insidioso de los síntomas en muchos pacientes o, como se ha visto en series de grandes familias, un tercio de los pacientes son asintomáticos al momento del diagnóstico ${ }^{1}$. El signo de Beevor, consistente en la elevación del ombligo al contraer los músculos rectos abdominales, debido a una debilidad mayor de los rectos abdominales inferiores y considerado característico en la DMFEH, se observó solo en 2 de nuestros pacientes ${ }^{22}$. Este signo es también observado en otras patologías neurológicas, incluidas otras miopatías ${ }^{23}$. Se ha ensayado el uso de clenbuterol y anabólicos en la DMFEH que produciría un aumento de la masa muscular, pero esto no se asocia a un mejor desempeño funcional o incluso de la fuerza, por lo tanto, no fueron usados en nuestros pacientes ${ }^{24-26}$.

En suma, esta pequeña serie permite plantear los problemas inherentes al diagnóstico y manejo de la DMFEH, así como también discutir su patogenia. El manejo de la DMFEH es esencialmente sintomático y los tratamientos farmacológicos están lejos de ser una ayuda real al manejo de esta u otras distrofias, pero el estudio genético es una herramienta fundamental porque permite un diagnóstico claro, que proporciona tranquilidad al paciente y su familia, y permite consejo genético con toda la información disponible.

\section{Referencias}

1. Padberg G. Facioscapulohumeral disease. Thesis. University of Leiden; 1982. 
2. Flanigan KM, Coffeen CM, Sexton L, Stauffer D, Brunner S, Leppert MF. Genetic characterization of a large, historically significant Utah kindred with facioscapulohumeral dystrophy. Neuromuscular disorders : NMD 2001; 11 (6-7): 525-9.

3. Tawil R, Van Der Maarel SM. Facioscapulohumeral muscular dystrophy. Muscle \& nerve 2006; 34 (1): 1-15.

4. Hassan A, Jones LK, Milone M, Kumar N. Focal and other unusual presentations of facioscapulohumeral muscular dystrophy. Muscle \& nerve 2012; 46 (3): 421-5.

5. Laforêt P, de Toma C, Eymard B, Becane HM, Jeanpierre M, Fardeau M, et al. Cardiac involvement in genetically confirmed facioscapulohumeral muscular dystrophy. Neurology 1998; 51 (5): 1454-6.

6. Padberg GW, Lunt PW, Koch M, Fardeau M. Diagnostic criteria for facioscapulohumeral muscular dystrophy. Neuromuscular disorders : NMD 1991; 1 (4): 231-4.

7. Ricci E, Galluzzi G, Deidda G, Cacurri S, Colantoni L, Merico B, et al. Progress in the molecular diagnosis of facioscapulohumeral muscular dystrophy and correlation between the number of KpnI repeats at the $4 \mathrm{q} 35$ locus and clinical phenotype. Annals of neurology 1999; 45 (6): 751-7.

8. Deidda G, Cacurri S, Piazzo N, Felicetti L. Direct detection of $4 \mathrm{q} 35$ rearrangements implicated in facioscapulohumeral muscular dystrophy (FSHD). Journal of medical genetics 1996; 33 (5): 361-5.

9. Gabellini D, Green MR, Tupler R. Inappropriate gene activation in FSHD: a repressor complex binds a chromosomal repeat deleted in dystrophic muscle. Cell. 2002; 110 (3): 339-48.

10. Tassin A, Laoudj-Chenivesse D, Vanderplanck C, Barro $\mathrm{M}$, Charron S, Ansseau E, et al. DUX4 expression in FSHD muscle cells: how could such a rare protein cause a myopathy? Journal of cellular and molecular medicine. 2013; 17 (1): 76-89.

11. Lemmers RJLF, van der Vliet PJ, Klooster R, Sacconi S, Camaño P, Dauwerse JG, et al. A unifying genetic model for facioscapulohumeral muscular dystrophy. Science (New York, N.Y.) 2010; 329 (5999): 1650-3.

12. Tim RW, Gilbert JR, Stajich JM, Rampersaud E, Viles $\mathrm{KD}$, Tawil R, et al. Clinical Studies in Non-chromosome 4-Linked Facioscapulohumeral Muscular Dystrophy. Journal of clinical neuromuscular disease 2001; 3 (1): $1-7$.

13. Orrell RW, Tawil R, Forrester J, Kissel JT, Mendell JR, Figlewicz D a. Definitive molecular diagnosis of facioscapulohumeral dystrophy. Neurology 1999; 52 (9): 1822-6.

14. Wijmenga C, Brouwer OF, Padberg GW, Frants RR. Transmission of de-novo mutation associated with facioscapulohumeral muscular dystrophy. Lancet 1992; 340 (8825): 985-6.

15. Zatz M, Marie SK, Passos-Bueno MR, Vainzof M, Campiotto S, Cerqueira a, et al. High proportion of new mutations and possible anticipation in Brazilian facioscapulohumeral muscular dystrophy families. American journal of human genetics 1995; 56 (1): 99-105.

16. Morton NE, Chung CS. Formal genetics of muscular dystrophy. American journal of human genetics 1959; 11: 360-79.

17. Tawil R, Forrester J, Griggs RC, Mendell J, Kissel J, McDermott $M$, et al. Evidence for anticipation and association of deletion size with severity in facioscapulohumeral muscular dystrophy. The FSH-DY Group. Annals of neurology 1996; 39 (6): 744-8.

18. Butz M, Koch MC, Müller-Felber W, Lemmers RJLF, van der Maarel SM, Schreiber H. Facioscapulohumeral muscular dystrophy. Phenotype-genotype correlation in patients with borderline D4Z4 repeat numbers. Journal of neurology 2003; 250 (8): 932-7.

19. Arahata K, Ishihara T, Fukunaga H, Orimo S, Lee JH, Goto K, et al. Inflammatory response in facioscapulohumeral muscular dystrophy (FSHD): immunocytochemical and genetic analyses. Muscle \& nerve. Supplement 1995; (2): S56-66.

20. Figarella-Branger D, Pellissier JF, Serratrice G, Pouget J, Bianco N. Immunocytochemical study of the inflammatory forms of facioscapulohumeral myopathies and correlation with other types of myositis. Annales de pathologie 1989; 9 (2): 100-8.

21. Lemmers RJLF, O’Shea S, Padberg GW, Lunt PW, van der Maarel SM. Best practice guidelines on genetic diagnostics of Facioscapulohumeral muscular dystrophy: workshop 9th June 2010, LUMC, Leiden, The Netherlands. Neuromuscular disorders : NMD 2012; 22 (5): 463-70.

22. Awerbuch GI, Nigro MA, Wishnow R. Beevor's sign and facioscapulohumeral dystrophy. Archives of neurology. 1990; 47 (11): 1208-9.

23. Hilton-Jones D. Beevor's sign. Practical neurology 2004; (4): 176-7.

24. Oya Y, Ogawa M, Kawai M. Therapeutic trial of beta 2-adrenergic agonist clenbuterol in muscular dystrophies. Rinshō shinkeigaku = Clinical neurology 2001; 41 (10): 698-700.

25. Kissel JT, McDermott MP, Natarajan R, Mendell JR, Pandya S, King WM, et al. Pilot trial of albuterol in facioscapulohumeral muscular dystrophy. FSH-DY Group. Neurology 1998; 50 (5): 1402-6.

26. Rose MR, Tawil R. Drug treatment for facioscapulohumeral muscular dystrophy. The Cochrane database of systematic reviews 2004; (2): CD002276. 